\title{
Numerical Simulation and Experimental Study on Liquid Holding Capacity of Eeddy Current Tools
}

\author{
Xiaolei Du, JihongTian ${ }^{*}$ and Fengsheng Yao \\ Offshore Oil Tower, 388 Tongxie Rd. 200335 Shanghai, China
}

\begin{abstract}
In view of the problems existing in the field application of eddy current tools, the research on eddy current tools drainage gas recovery was carried out.Based on Fluent software and ground experiments, the optimal structure size of the eddy current drainage gas production tool is determined as follows: the wing height of the helix is $1.5 \mathrm{~mm}$, the diameter of the helix is $50 \mathrm{~mm}$, the wing width of the helix is $2 \mathrm{~mm}$, and the helix Angle is $55^{\circ}$, which provides reference for field application.
\end{abstract}

\section{Introduction}

Eddy-current drainage gas recovery is a new technology emerging in the 21 st century. It is one of the new technology research projects of low-yielding oil fields funded by the U.S. Department of Energy in 2001.How it works[1-2]Is when there is no rule of gas - liquid two phases turbulent flow after entering eddy current tools, eddy current tools make fluid rapidly rotating, the acceleration makes the heavier liquid into the wall, the fluid flow upward movement along the tool, the structure of the tools to achieve ideal state, the rotation of the fluid into two phase laminar helical flow of the rules of the high efficiency of spiral Angle can spread and maintain a very long distance, can also carry more much of the liquid, so as to improve the ability of gas well drainage gas recovery.

Orthogonal experimental design method is a mathematical method which uses orthogonal tables to arrange multi-factor experiments and makes use of probability theory, mathematical statistics and practical experience to carry out data analysis[3]. In the study of the drainage gas recovery effect of eddy current tools, multiple structural and size factors affect the evaluation indexes. Due to the large number and level of factors, the number of tests will also be large, which not only brings a great amount of work to the research, but also wastes resources and time. In this paper, through orthogonal test, the idea is to use partial test instead of full test, select a representative test scheme to carry out the test, through the representative test results analysis, understand the full test situation, determine the best eddy current tool structure size[4].

\footnotetext{
* Corresponding author: tianjh2@cnooc.com.cn
} 


\section{Physical model}

According to the field application of eddy current tools ${ }^{[5]}$ Physical model A and simulation calculation area model B were established, as shown in Figure 1.

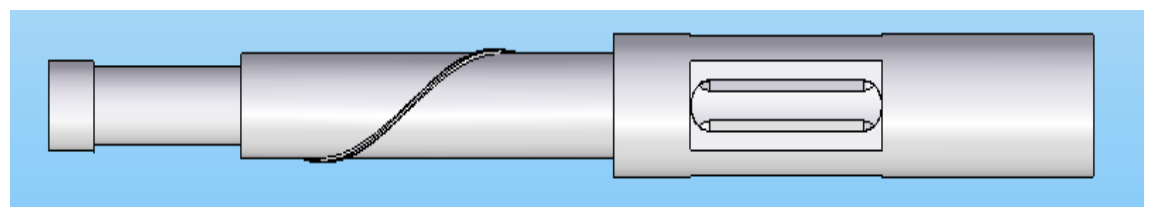

a) Physical model

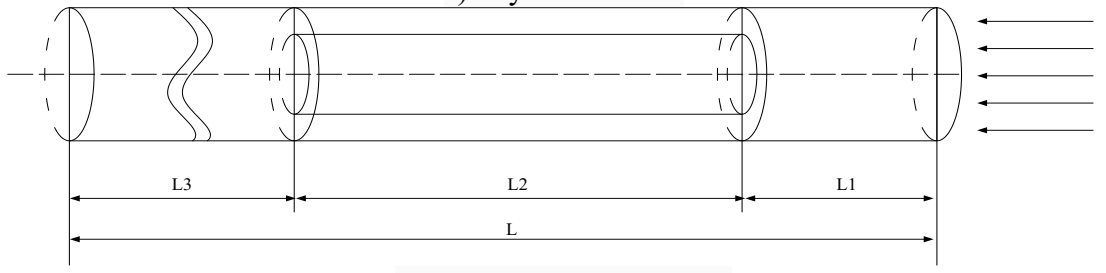

b) Computational domain

Fig. 1. Tubing calculation model with eddy current tool installed

\section{Working principle of eddy current tool}

For gas Wells, gas-liquid two-phase flow is involved, and eddy current tools transform the gas-liquid two-phase flow from a turbulent flow state to a stable spiral stratified flow state. The helical stratified flow can reduce the loss of fluid slippage, increase the pressure difference in bottom hole production and increase the liquid carrying rate of gas, so as to increase the production of gas well.

\section{Numerical calculation and result analysis}

\subsection{Numerical simulation of boundary conditions and solution methods}

Inlet boundary conditions: gas phase was selected as continuous phase and liquid phase as discrete phase. The inlet is the velocity inlet boundary, and the inlet velocity of each phase is set as $/ \mathrm{s} .1 \mathrm{mF}$ or the liquid phase, when the entry boundary condition is set, the volume fraction is 0.0005 . Outlet boundary condition: both continuous phase and discrete phase choose sufficient outflow boundary condition. Wall conditions: the standard wall function is selected to deal with the wall boundary layer, and the wall boundary velocity and mass are not added. The default setting of the system is maintained.In the numerical simulation based on Fluent software, the gas-liquid two-phase fluid was set to pass through the eddy current tool, and the three-dimensional unsteady turbulent flow was carried out in the tubing from the bottom to the top.The standard $\kappa-\varepsilon$ turbulence model and Mixture model of multiphase flow were selected to solve the gas-liquid separation model through eddy current tool by SIMPLE algorithm. The simulation calculation is limited by the number of grids and the computer memory, and the length of the simulated tubing is $2000 \mathrm{~mm}$.

\subsection{Numerical calculation}

To verify the key structural dimensions in the eddy current tool, as shown in Fig. 2, H is the 
wing height of the helix, D is the diameter of the helix, $\alpha$ is the helix Angle, and B is the wing width of the helix.In this paper, the difference of liquid holdup between inlet and outlet of the model was selected as the evaluation index to analyze their influence on drainage gas recovery effect. The changes of liquid holdup difference under the following conditions were calculated respectively to determine the optimal structure size of the eddy current tool.
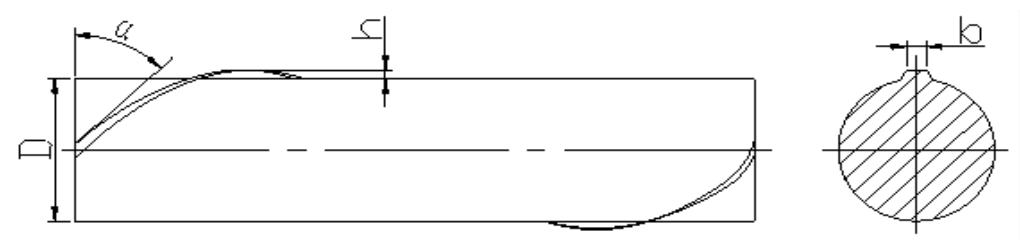

Fig. 2. Structure size diagram

\subsubsection{Influence of the height of the spirochete wing on liquid holdup}

The inlet velocity was $1 \mathrm{~m} / \mathrm{s}$, the liquid volume fraction was 0.0005 , the diameter of the helix was $40 \mathrm{~mm}$, the wing width of the helix was $2 \mathrm{~mm}$, the helix Angle was $55^{\circ}$, and the wing height of the helix was $1.5 \mathrm{~mm}, 3 \mathrm{~mm}, 5 \mathrm{~mm}, 7 \mathrm{~mm}$, and $10 \mathrm{~mm}$. When the helix wing height is changed, the liquid holdup difference between inlet and outlet is shown in Fig. 3.

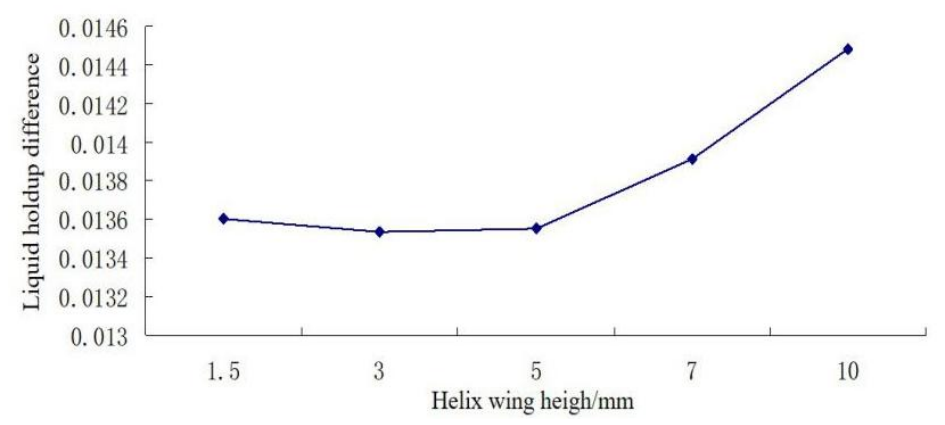

Fig. 3. Variation of liquid holdup difference corresponding to wing height

As can be seen from Fig. 3, with the change of wing height, the difference of liquid holdup firstly decreases and then increases. When the wing height is $3 \mathrm{~mm}$, the difference of liquid holdup is the minimum of 0.13533 . When the wing height is greater than $5 \mathrm{~mm}$, the slope of the curve is large, which indicates that the wing height of the helix in the eddy tool has a great influence on the drainage gas recovery effect.

\subsubsection{Influence of spirochete diameter on liquid holdup}

The inlet velocity is $1 \mathrm{~m} / \mathrm{s}$, the liquid volume fraction is 0.0005 , the width of the helix wing is $2 \mathrm{~mm}$, the height of the helix wing is $1.5 \mathrm{~mm}$, the helix Angle is $55^{\circ}$, and the diameter of the helix is $35 \mathrm{~mm}, 40 \mathrm{~mm}, 45 \mathrm{~mm}, 50 \mathrm{~mm}$ and $55 \mathrm{~mm}$. When the diameter of the spirochete was changed, the liquid holdup difference between import and export was shown in Fig. 4. 


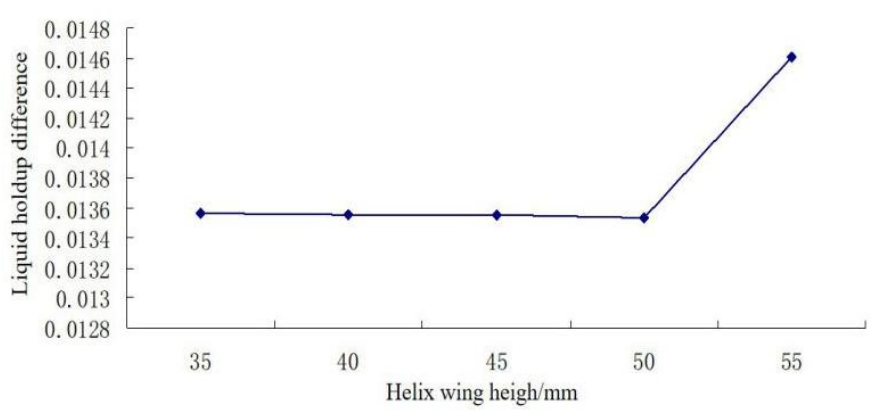

Fig. 4. Variation of liquid holdup difference corresponding to diameter

As can be seen from Fig. 5, when the diameter of the helix is between 35 and $50 \mathrm{~mm}$, the liquid holdup difference curve has little change trend, and the optimal value is $50 \mathrm{~mm}$. However, when the diameter exceeds $50 \mathrm{~mm}$, the slope of the curve becomes larger, indicating that the diameter of the helix has a great influence on the drainage gas recovery effect of the eddy current tool.

\subsubsection{Influence of wing width of the spirochete on liquid holdup}

The inlet velocity was $1 \mathrm{~m} / \mathrm{s}$, the liquid volume fraction was 0.0005 , the diameter of the helix was $40 \mathrm{~mm}$, the height of the helix wing was $1.5 \mathrm{~mm}$, the helix Angle was $55^{\circ}$, and the wing width of the helix was $2 \mathrm{~mm}, 3 \mathrm{~mm}, 5 \mathrm{~mm}, 7 \mathrm{~mm}$, and $9 \mathrm{~mm}$. When the wing width of the helix is changed, the liquid holdup difference between inlet and outlet is shown in Fig. 5.

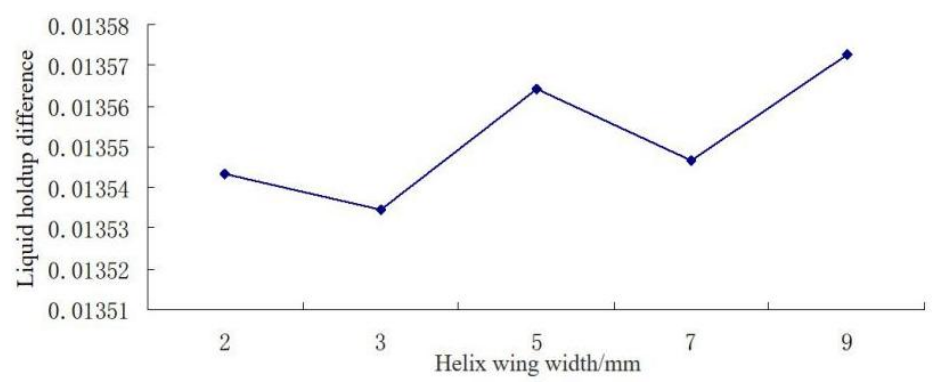

Fig. 5. Variation of liquid holdup difference corresponding to wing width

As can be seen from Fig. 5, with the change of wing width, the difference curve of liquid holdup firstly decreased and then increased, and then increased after the decrease. When the wing width is $3 \mathrm{~mm}$, the difference of liquid holdup reaches the minimum value of 0.135345 . It can be seen from the changing trend of the curve that the wing width has an effect on the discharge effect of the eddy current tool.

\subsubsection{Influence of helix Angle on liquid holdup}

The inlet velocity was $1 \mathrm{~m} / \mathrm{s}$, the liquid volume fraction was 0.0005 , and the helix Angle was changed. The diameter of the helix was $40 \mathrm{~mm}$, the height of the helix wing was $1.5 \mathrm{~mm}$, and the width of the helix wing was $2 \mathrm{~mm}$. The helix Angle was $35^{\circ}, 40^{\circ}, 45^{\circ}, 50^{\circ}, 55^{\circ}$, and the liquid holding ratio difference between the inlet and outlet was shown in Fig. 6. 


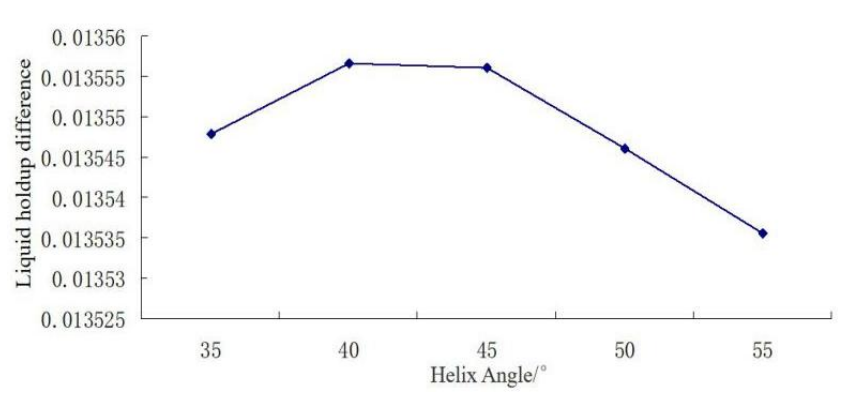

Fig. 6. Variation of liquid holdup difference corresponding to helix Angle

As can be seen from Fig. 6, with the increase of helical Angle, the liquid holdup difference curve firstly increases and then decreases. When the helical Angle is $55^{\circ}$, the liquid holdup difference reaches the minimum value of 0.135356 . This shows that the helix Angle in the vortex tool has a great influence on drainage gas recovery.

\section{Laboratory experiment of eddy current drainage gas recovery}

A set of laboratory simulation experimental equipment was designed to verify the influence of eddy current structure parameters on liquid holdup based on numerical simulation.

\subsection{Experimental device design}

The schematic diagram of the indoor experimental device for eddy current drainage gas production is shown in Figure 7, which is mainly composed of gas supply system, liquid supply system, simulated pipe string system, gas-liquid separation system, data acquisition system and other major parts.

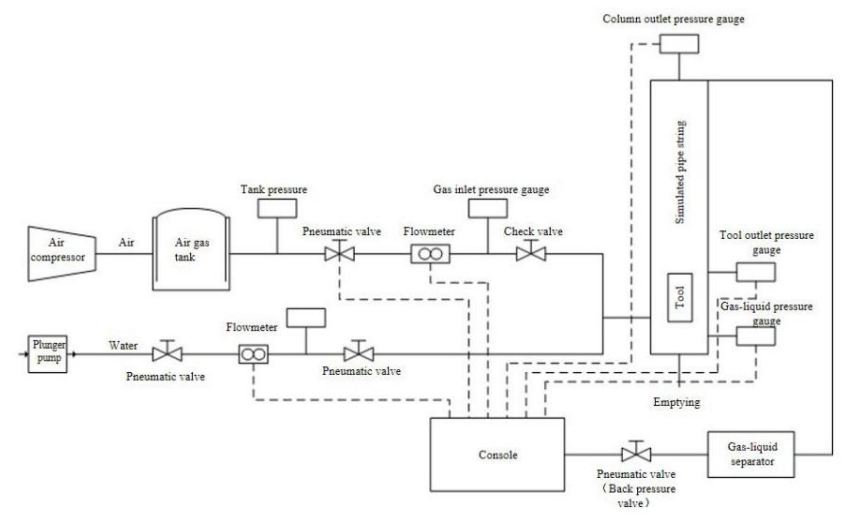

Fig. 7. Schematic diagram of experimental equipment

\subsection{Experimental analysis}

Under the experimental condition of $0.1 \mathrm{MPa}$ pressure, the effects of drainage gas recovery with two different structure sizes of eddy current tools are simulated respectively.Figure 8 shows the flow pattern of gas-liquid two-phase flow in the pipe string simulated before and after loading the vortex tool. It can be clearly seen that after loading the vortex tool, the 
flow pattern changes from turbulent flow pattern to a flow pattern in which the gas phase moves upward along the center of the pipe string and the liquid phase moves upward along the pipe wall in a spiral way.The experimental results of two tools with different structural sizes are shown in Table 1. Tool 1 is: $\mathrm{D}=50 \mathrm{~mm}, \alpha=55^{\circ}, \mathrm{H}=1.5 \mathrm{~mm}, \mathrm{~B}=2 \mathrm{~mm}$; Tool 2 is: $\mathrm{D}=40 \mathrm{~mm}, \alpha=55^{\circ}, \mathrm{H}=3 \mathrm{~mm}, \mathrm{~B}=2 \mathrm{mmQg}$ - gas flow rate, $\mathrm{Kg} / \mathrm{min}$; Qw- liquid flow, $\mathrm{Kg} / \mathrm{min} ; \mathrm{QW} /(\mathrm{QW}+\mathrm{QG})$ - liquid holdup.
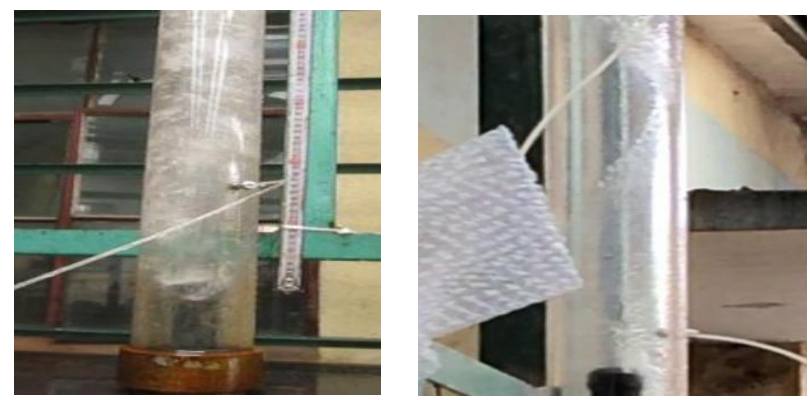

Fig. 8. Experimental flow pattern

Table 1. Experimental results

\begin{tabular}{|c|c|c|c|}
\hline The name of the & $\mathbf{L o g}(\mathbf{Q g})$ & $\mathbf{Q w}$ & $\mathbf{Q w} / \mathbf{Q w}+\mathbf{Q g})$ \\
\hline \multirow{3}{*}{ Tool 1 } & 0.5635 & 0.114 & 0.0302 \\
\cline { 2 - 4 } & 0.5682 & 0.3745 & 0.0919 \\
\cline { 2 - 4 } & 0.5705 & 1.4275 & 0.2773 \\
\cline { 2 - 4 } & 0.5736 & 2.8445 & 0.4317 \\
\hline \multirow{4}{*}{ Tool 2 } & 0.6042 & 0.3765 & 0.0856 \\
\cline { 2 - 4 } & 0.6058 & 0.7115 & 0.1499 \\
\cline { 2 - 4 } & 0.6064 & 2.1975 & 0.3517 \\
\cline { 2 - 4 } & 0.6075 & 3.561 & 0.4679 \\
\hline
\end{tabular}

According to the difference value of liquid holdup in the evaluation index during numerical simulation, the liquid holdup curves of the two tools were drawn based on the experimental results with $\log (\mathrm{Qg})$ as the abscisson and $\mathrm{Qw} /(\mathrm{Qw}+\mathrm{Qg})$ as the ordinate, as shown in Fig. 9.It can be seen that the liquid holdup capacity increases with the increase of Qg.By comparing the liquid holdup curves of the two tools, Tool 1 was able to carry liquid when the gas volume was low. The correctness of the numerical simulation is further illustrated.

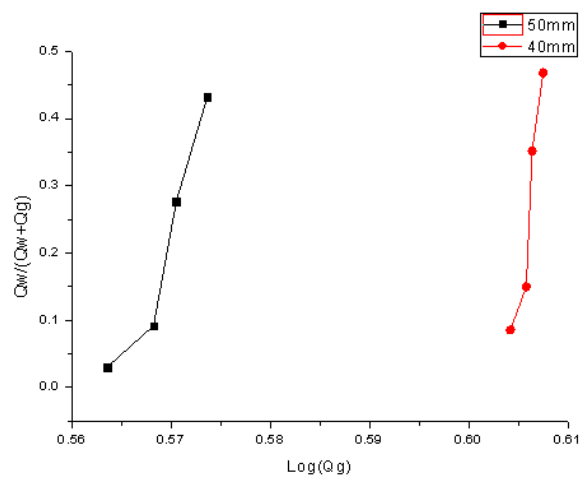

Fig. 9. Curve of liquid holdup 


\section{Conclusion}

According to the field application, the physical model of the eddy current tool is established. Aiming at the problems of eddy current tool in drainage gas recovery, several structural factors of the tool that may affect the drainage effect are analyzed in this paper.Through calculation and comparison, the following conclusions are drawn.

(1) The structural factors of the helix of eddy current tools have a great influence on the drainage effect. Through comparative analysis, the optimal value range of each factor is obtained as follows: the wing height of the helix is $1.5-3 \mathrm{~mm}$, the diameter of the helix is $50 \mathrm{~mm}$, the wing width of the helix is $2-3 \mathrm{~mm}$, and the helix Angle is $55^{\circ}$.

(2) Based on the results of numerical simulation and through the ground experiment of eddy current drainage gas production, the optimal structural size of eddy current tool is determined. The wing height of the helix is $1.5 \mathrm{~mm}$, the diameter of the helix is $50 \mathrm{~mm}$, the wing width of the helix is $2 \mathrm{~mm}$, and the helix Angle is $55^{\circ}$, which provides a reliable reference for field application.

(3) It is recommended to further study the optimal value of each factor combination, carry out field tests of eddy current tools, verify the reliability of numerical simulation and experimental results, and provide theoretical support for field application.

\section{References}

[1] Meher Surendra,Gioia Falcone, and Catalin Teodoriu,Invenstigation of Swirl Flows Applied to the Oil and Gas Industry[J]. paper SPE 115938 presented at the 2008 SPE Annual Technical Conference andExhibition held in , 21-24 September 2008. Denver, Colorado, USA

[2] Ali A J, Scott S L, Fehn B J. Investigation of New Tool to Unload Liduids from Stripper-Gas Wells[C]. paper 84136-MS presented at the SPE Annual Technical Conference and Exhibition ,5-8 October 2003, : SPE, 2003. Denver, Colorado

[3] Orthogonal experimental design method [M]. Shanghai: Shanghai Science and Technology Press, 1979.

[4] Chang Guojun, Huang Kun, Ao Feng, Wang Xiang.Application of eddy current drainage gas recovery technology in offshore gas fields [J]. China Petroleum \& Petrochemical,2017(05):39-40.

[5] Chen Jie, Yang Zhi, Geng Fengkang, Chen Yong, Kang Lu.Optimization of Structural Parameters of Downhole Eddy Current Tools [J]. Science Technology and Engineering,2020,20(30):12385-12396. 